\title{
The LUCA project - Laser and Ultrasound Co-Analyzer for Thyroid Nodules: Overview and Current Status
}

\author{
L. Cortese $^{1 *}$, G. Aranda ${ }^{2}$, M. Buttafava ${ }^{3}$, D. Contini ${ }^{4}$, A. Dalla Mora ${ }^{4}$, S. de Fraguier5, H. Dehghani ${ }^{6}$, E. \\ Garcia7, R. Gomis ${ }^{2}$, F. Hanzu ${ }^{2}$, K. Krischak ${ }^{8}$, G. Lo Presti ${ }^{1}$, M. Mora ${ }^{2}$, A. Pifferi ${ }^{4}$, M. Renna ${ }^{3}$, B. Rosinski ${ }^{9}$, S. \\ Konugolu Venkata Sekar ${ }^{4}$, M. Squarcia ${ }^{2}$, P. Taroni ${ }^{4}$, A. Tosi ${ }^{3}$, U. M. Weigel ${ }^{7}$, S. Wojtkiewicz ${ }^{6}$, P. Zolda $^{8}$, T. \\ Durduran ${ }^{1,10 *}$ \\ 1 ICFO-Institut de Ciències Fotòniques, The Barcelona Institute of Science and Technology, 08860 Castelldefels (Barcelona), Spain \\ 2 IDIBAPS, Fundació Clínic per la Recerca Biomèdica, Barcelona, Spain \\ 3 Politecnico di Milano-Dipartimento di Elettronica, Informazione e Bioingegneria, Milan, Italy \\ 4 Politecnico di Milano-Dipartimento di Fisica, Milan, Italy \\ 5 ECM - ECHO CONTROL MEDICAL, S.A.S., Angoulême, France \\ 6 University of Birmingham, United Kingdom \\ 7 HemoPhotonics S.L., Castelldefels (Barcelona), Spain \\ 8 EIBIR, The European Institute for Biomedical Imaging Research, Vienna, Austria \\ 9 VERMON S.A., Tours, France \\ 10 Institució Catalana de Recerca i Estudis Avançats (ICREA), 08015 Barcelona, Spain \\ *Authors list is in alphabetic order except for presenting author and project coordinator \\ Author e-mail address: lorenzo.cortese@icfo.es
}

\begin{abstract}
We present the current status of the LUCA-project whose aim is to develop an innovative device combining ultrasound and diffuse optics for an improved screening of the thyroid cancer.

OCIS codes: (170.0170) Medical Optics and Biotechnology; (170.6935) Tissue characterization; (170.4580) Optical diagnostics for medicine; (170.3890) Medical Optics Instrumentation;
\end{abstract}

\section{Introduction}

Thyroid cancer is a major and growing health challenge with around three hundred thousand new cases diagnosed worldwide annually. The poor screening quality of current methods and the high prevalence of thyroid nodules do not provide sufficient support to surgeons in their decision on the appropriate course of action, which leads to a significant number of unnecessary surgeries and a reduced quality of life for patients. This calls for new modalities and biomarkers to be introduced for an increased sensitivity and specificity of the screening process.

The LUCA project ${ }^{1}$ aims to tackle this need by producing a point-of-care device for the screening of thyroid nodules. The device is based on near-infrared diffuse correlation spectroscopy (DCS) [1] and time-resolved spectroscopy (TRS) [2] combined with an ultrasound system (US) and a probe that enables multimodal data acquisition. LUCA device will allow the retrieval at the same time of information about tissue hemodynamics (microvascular blood flow, blood oxygenation), chemical constitution (scattering spectrum, collagen, water and lipid concentrations) as well as anatomy. This may overcome the shortcomings of present techniques assuring a better screening for malignant nodules. LUCA-project involves a multidisciplinary team of eight partners, including end-users in endocrinology and radiology, three research groups in the field of optics, opto-electronics and biophotonics and industry players with a background in development of innovative photonic and ultrasonic components and medical devices. The first phase of the project focused on the development and construction of new optoelectronic components, while the second phase on implementation and clinical validation of the LUCA demonstrator.

We will present the current status of the LUCA-project (24 months, mid-point). The first prototype of the LUCA device will be presented, together with a detailed description of each newly developed sub-system. In particular we will focus on the validation of the new - lower cost and increased quality - components and the sub-systems.

\section{Concept}

The goal of the thyroid screening is to identify high-risk nodules for malignancy, for further, more invasive evaluation and potentially a surgical intervention, and to avoid missing any malignant nodules. After an initial ultrasound screening, which is poor in sensitivity and in specificity, depending on the suspected type of the nodule, the most common tool to test malignancy is ultrasound-guided fine needle aspiration biopsy (FNAB) of the suspicious nodule. However, the effectiveness of the FNAB in thyroid cancer is also limited. A large number of nondiagnostic and/or false positive FNAB results lead to unnecessary surgeries [3]. The high prevalence of thyroid nodules (up to $76 \%$ of the population) implies that even modest improvement in strategies to characterize lesions could have a great impact - not only in term of better therapeutic outcomes, but also in terms of a more efficient use

\footnotetext{
${ }^{1}$ http://www.luca-project.eu/
} 
of health resources. Figure 1 shows the short and long-term advances proposed by LUCA. Our aim is to delineate a procedure that permits to better distinguish between benign and malignant nodules, reducing the number of FNAB and unnecessary surgeries.

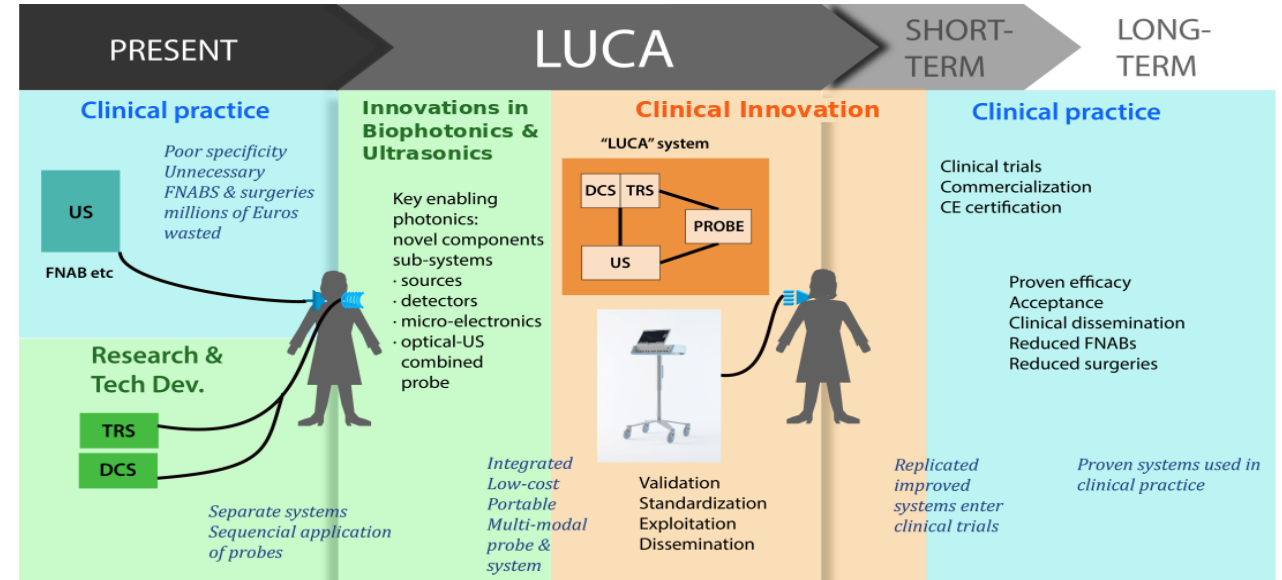

Figure 1 Overview of the LUCA vision starting at the present clinical practice in thyroid screening and current research followed by the proposed innovations during the LUCA period. From http://www.luca-project.eu .

\section{Device}

There are four main technologies involved in the project: US, DCS, TRS and the multimodal probe. Furthermore, the LUCA device will exploit a dedicated module for the data analysis.

-The Ultrasound technology is the current standard technology used in the screening of thyroid cancer. For this technology the focus is on the innovation challenges involved in the integration of the extra information provided by the optical module and the new hybrid probe. In this respect a specific software interface was developed to exchange information with the optical subsystem.

-DCS and TRS have reached commercial-level maturity as independent solutions. TRS is used for the determination of chromophore concentrations such as oxy/deoxy-hemoglobin, water, lipid and collagen [2]. The DCS system measures deep tissue micro-vascular blood flow [1]. The LUCA goal in relation to these technologies is to develop suitable compact, low-cost solutions at component and subsystem level, and integrate them efficiently in a hybrid stand-alone photonics module. At this stage of the project all the LUCA optical subsystems have been developed, tested and validated with standard phantoms. The DCS laser subsystem developed consists of a fiber coupled laser diode (wavelength $785 \mathrm{~nm}, 120 \mathrm{~mW}$ maximum power) with custom driving and thermo-electric cooling electronics. This solution allows a reduction of the costs of 10-15 times with respect of a standard DCS laser system. The DCS detection system provides up to 16 independent detection channels (QE @785 nm > 55\%; maximum dark count rate $1000 \mathrm{c} / \mathrm{s}$; timing resolution $1 \mathrm{~ns}$ ) connected to a custom developed FPGA based 16channels correlator. The developed correlator allows a reduction of the costs of roughly 5 times with respect to standard commercial correlators. The TRS sub-system exploits eight fiber coupled edge emitting diodes (average

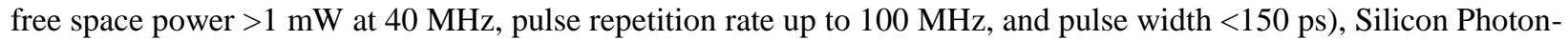
multipliers (SiPM) detectors, and a time-correlated single photon counting system based on a Time-to-Digital Converter (TDC). Finally, the cost for all TRS sub-systems will be about five times lower than commerciallyavailable equivalent systems.

-The hybrid optics/US probe is thought to acquire both types of data and to deliver them independently to each module. The first phase of the project carried out the construction of a fully functional initial evaluation system that mimics the hardware, software and the sensor.

-Data analysis will have a dedicated module inside LUCA device consisting in a computer with a dedicated graphic processing unit (GPU) for calculations. A NIRFAST [5] toolbox for TRS has been implemented, while a NIRFAST toolbox for DCS has been completely developed for the LUCA project. The data analysis system will process the output provided by LUCA device to calculate the nodule hemodynamics and components.

\section{Acknowledgement}

The research was founded by LUCA (grant agreement no. 688303, H2020-ICT-2015). The project received furtherfinancial support from Fundació CELLEX Barcelona, the "Severo Ochoa" Programme for Centres of Excellence in R\&D (SEV-2015-0522), the Obra social "la Caixa" Foundation (LlumMedBcn).

[1] T. Durduran (2010) Rep. Prog. Phys.73:076701.

[2] M. Patterson (1989) Appl. Opt. 28, 2331

[3] Rago et al (2007) Clin.Endocrinol.(Oxf) 66: 13-20.

[4] Linder et al. (2016) PLoS ONE:10.1371/0147851.

[5] H. Dehghani et al. (2009) Comm. in Num. Meth. in Eng. 25:711-732 